\title{
Surgery to Oligometastatic Breast Cancer after Excellent Response to Palbociclib and Letrozole Therapy: Pitfall of Ultrasound Therapeutic Evaluation
}

\author{
Suzuka Fujii Shoji Oura Shinichiro Makimoto \\ Department of Surgery, Kishiwada Tokushukai Hospital, Kishiwada, Japan
}

\section{Keywords}

Breast cancer · CDK 4/6 inhibitor · Oligometastasis · Sarcoid-like reaction

\begin{abstract}
A 48-year-old woman with regional recurrences of breast cancer in the axillar and supraclavicular regions was referred to our hospital. Under the diagnosis of recurrent luminal breast cancer with a high Ki-67 labeling index of $>30 \%$ and a disease-free interval of 13 years, the patient began to receive palbociclib, letrozole, and luteinizing hormone-releasing hormone agonist, resulting in marked response of the supraclavicular lesion and stable disease of the axillar lesion on ultrasound (US) evaluation. Positron emission tomography (PET)/computed tomography of the axillar and supraclavicular foci showed high and no avidities before and after treatment, respectively. The unmovable neck lesion became movable with the treatment. The patient, therefore, underwent surgical resection of the 2 metastatic foci to examine the discordant therapeutic efficacy against the 2 metastatic foci on 2 image modalities, that is, US and PET, and to possibly get a cure of the breast cancer oligometastasis. Pathological examination showed marked fibrosis and scant cancer cell residuals with microcalcifications in the neck tumor and massive sarcoid-like reaction with scant cancer cell residuals in the axillary nodes. The residual cancer cells showed estrogen and progesterone receptor positivities, human epidermal growth factor receptor type 2 negativity, and an extremely low Ki-67 labeling index of $2.5 \%$. The patient recovered uneventfully and has continued palbociclib-containing endocrine therapy for 1 year without any recurrences. Breast oncologists should well understand the basic principles of internal echo formation on US
\end{abstract}


and take the presence of sarcoid-like reaction in the cancer cell clusters into consideration on the therapeutic evaluation of metastatic breast cancer.

\section{Introduction}

Breast cancer often metastasizes to the locoregional tissue/lymph node, bone, lung, liver, and brain. Efficacy evaluation of some anticancer therapy against metastatic foci is mainly done with ultrasound, scintigraphy, magnetic resonance imaging, computed tomography (CT), and positron emission tomography/CT (PET/CT). Of these, ultrasonography (US) is frequently used to determine the therapeutic effect on locoregional recurrence from the viewpoint of its simplicity, noninvasiveness, and cost performance.

It is often observed that in a breast cancer patient with bone and liver metastases, bone metastasis continues to show favorable response to some anticancer therapy while the liver metastasis shows progressive disease. It, however, is extremely rare that one of the metastases in the same organ shows marked tumor shrinkage and the other shows obvious growth. The therapeutic effect on lymph node metastasis is generally the same regardless of the site of the metastatic lymph node. In addition, soft tissue recurrence also shows similar therapeutic efficacy to that of lymph node metastasis.

Supraclavicular lymph node metastasis is regarded as not local but regional metastasis, judged as stage IIIc breast cancer in case of no distant metastasis. Patients with stage IIIc breast cancer are generally the candidates not for immediate surgery but for primary systemic therapy followed by some kind of surgical intervention when the supraclavicular metastasis completely responds to the primary systemic therapy. Naturally, supraclavicular recurrence of breast cancer is generally out of the target for surgical intervention in daily clinical practice. However, regardless of the recurrent site, surgery to the oligometastatic foci with curative intent seems to be appropriate at least from the viewpoint of re-biopsy when the surgical intervention causes only nominal or fully acceptable harm to the patients. We herein report a case of breast cancer with metastatic foci in the axillar and supraclavicular regions showing discordant clinical responses to CDK 4/6 and aromatase inhibitors [1] on US and PET.

\section{Case Report}

A 48-year-old woman with regional recurrences of breast cancer in the axillar and supraclavicular regions was referred to our hospital. The patient had undergone bilateral nipple-preserving mastectomy, sentinel node biopsy, and immediate breast reconstruction using tissue expander followed by the replacement of silicone implant for her invasive ductal cancer 13 years before. After finishing adjuvant tamoxifen therapy for 5 years, the patient was lost to follow-up. At the re-visit to our hospital, we palpated an unmovable tumor in the right supraclavicular region and movable and swollen lymph nodes in the right axilla. PET/CT showed high avidities, that is, maximum standardized uptake value of up to 7.14, in the axillar and supraclavicular lesions (Fig. 1a). US showed a tumor with poorly defined margins, markedly attenuated posterior echoes, and heterogeneous low-level echoes in the supraclavicular lesion (Fig. 2a) and a well-defined and oval mass, $1.4 \mathrm{~cm}$ in size, with low and focal high internal echoes, and enhanced posterior echoes in the right axilla (Fig. 2b). Under the diagnosis of locoregional recurrences, core needle biopsy was done to the supraclavicular tumor. Histological examination of the tumor demonstrated 


\section{Case Reports in Oncology}

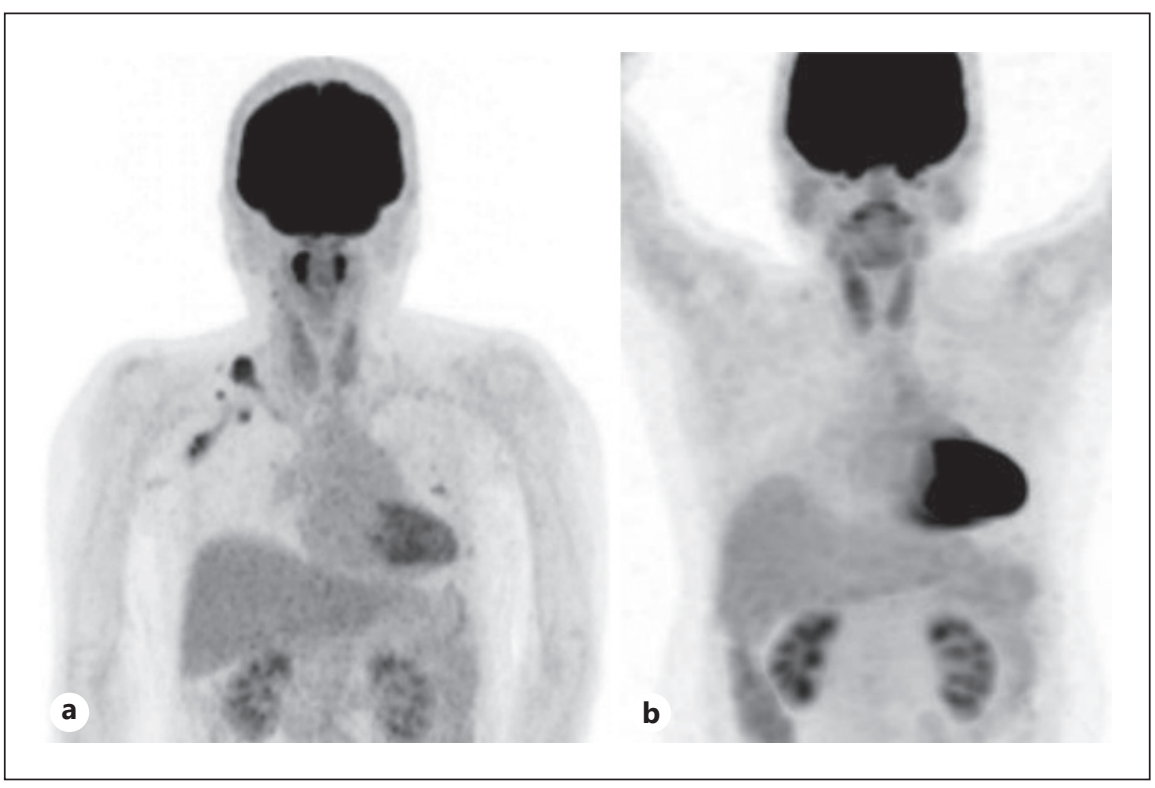

Fig. 1. a PET on recurrence showed marked avidities in the presumed recurrent foci ranging from the right axilla to the supreclavicular region. b PET after the treatment showed marked decrease of fluorodeoxyglucose accumulation in the right axilla and neck. PET, positron emission tomography.

atypical cells with a large nucleus and scant cytoplasm accompanied by lymphocyte infiltration (Fig. 3a). Immunohistochemical examination showed estrogen and progesterone receptor positivities, human epidermal growth factor receptor type 2 (HER2) negativity, and a high expression of Ki-67 labeling index, that is, $>30 \%$, leading to the diagnosis of luminal-type recurrent breast cancer. Due to the presence of an unresectable mass in the neck, the patient received combination therapy with palbociclib, that is, a CDK 4/6 inhibitor, luteinizing hormone-releasing hormone agonist, and letrozole, resulting in minor response for 15 months followed by sudden and marked tumor shrinkage of the neck tumor. US of the neck tumor showed complete disappearance of the presumed invasion to the dermis, a notable change of internal echoes from low to high, and clarification of the obscured bottom of the tumor (Fig. 2c), highly suggesting the neck tumor as becoming resectable. On palpation, dramatic change from unmovable to movable tumor was also detected. PET/CT showed no remarkable accumulations in both lesions after treatment (Fig. 1b). US of the axilla, however, showed no marked change in the presumed axillary lymph node recurrence (Fig. 2d). We, therefore, tried to resect the metastatic foci both for pathological evaluation and local control. Pathological examination showed marked fibrosis and scant cancer cell residuals with microcalcifications in the neck tumor (Fig. 3b) and massive sarcoid-like reaction with scant cancer cell residuals in the axillary nodes (Fig. 3c) The residual cancer cells showed estrogen and progesterone receptor positivities, HER2 negativity, and an extremely low Ki-67 labeling index of $2.5 \%$ (Fig. 3d). The patient recovered uneventfully and was discharged on the 7th day after operation. The patient has been well without any further recurrences for 12 months under palbociclibincluding therapy.

\section{Discussion}

Mammography depicts black and white areas when X-ray is irradiated toward the film through substances with low and high X-ray attenuation coefficients, for example, fat and calcification, respectively. On the other hand, US shows low and high internal echoes when the 


\section{Case Reports in Oncology}
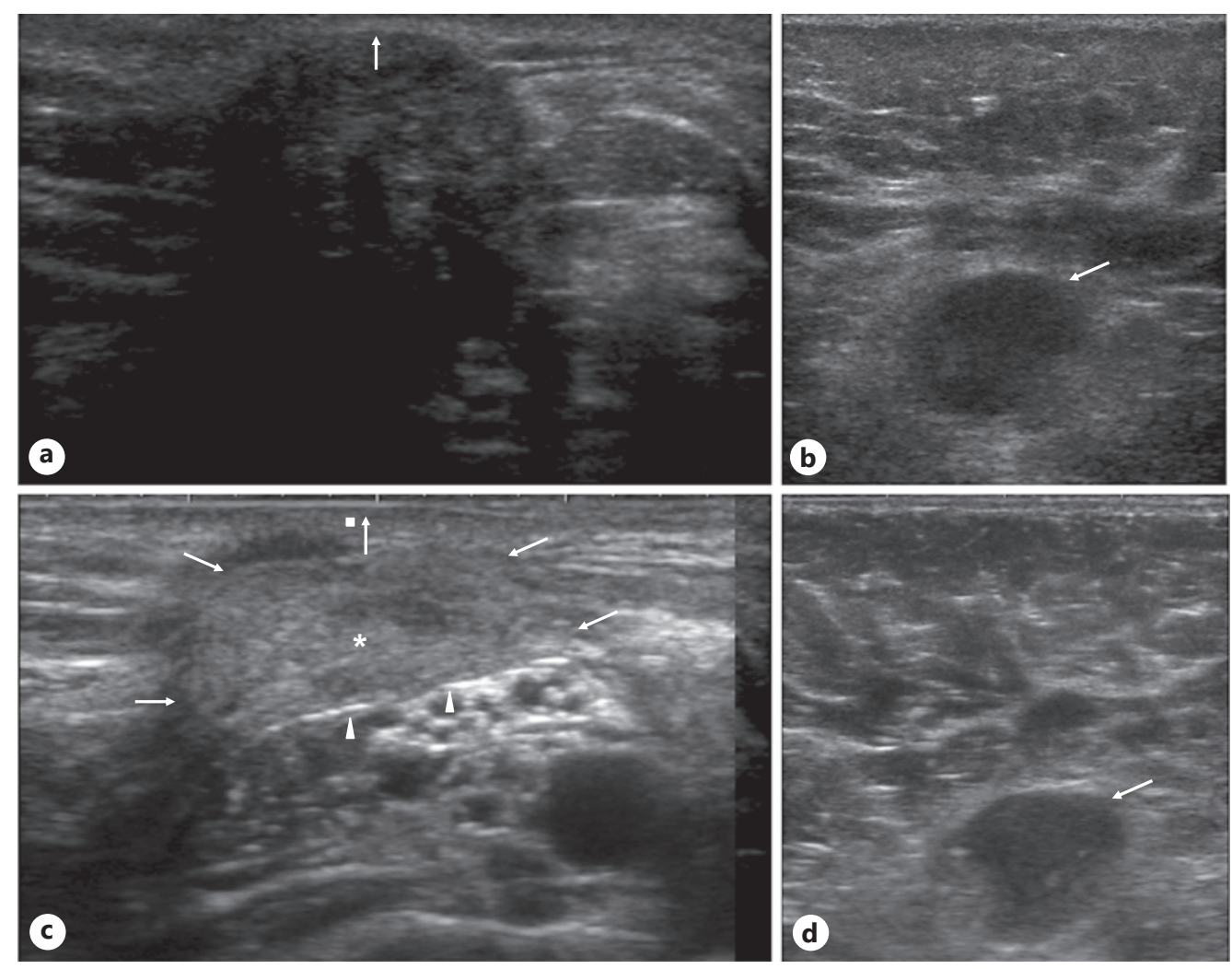

Fig. 2. US of the axillar and supraclavicular lesions. a US before treatment showed an oval mass with mixed low and high internal echoes, markedly attenuated posterior echoes, and presumed invasion to the dermis (arrow). b US before treatment showed a well-demarcated oval mass with predominantly low internal echoes and enhanced posterior echoes. c US after treatment showed marked tumor shrinkage (encompassed by arrows), a notable change of internal echoes from low to high echoes (asterisk), clarification of the obscured posterior margin before treatment (arrowheads), and clear detachment of the recurrent tumor from the dermis (square). d US after treatment showed similar findings to those of the axillar recurrent tumor before treatment. US, ultrasonography.

back scattering is weak and strong, respectively [2]. It is well known that back scattering becomes stronger when substances with different acoustic impedances are mixed, and backscattering becomes weaker when substances with similar acoustic impedances form some area. In this case, pathological findings showed treatment-induced fibrosis, microcalcifications, and scant cancer cell residuals in the neck tumor and massive sarcoid-like reaction, scant cancer cell residuals, and no fibrosis in the axillary nodes. These pathological findings well explain the discordance of treatment-induced changes on US findings between the axillary lymph nodes and the neck tumor.

Sarcoid-like reaction and sarcoidosis share a common T-cell-mediated pathway in the formation of granulomas. The former is considered to be induced by soluble antigenic factors released from the malignant cells and the latter by the host's reaction to some foreign substances $[3,4]$. We cannot speculate why sarcoid-like reaction occurred not in the neck tumor but in the axillary lymph nodes. Function of the lymph node as an immune organ might cause this difference. In addition, judged by the pathological anticancer effect of the neck tumor, scant cancer cells in the axillary lymph nodes should be the residuals of much larger cancer cell clusters, even though lacking treatment-induced fibrosis. The absence of fibrosis in the axillary lymph node might also be attributable to the character of lymph node as

\section{Karger's}



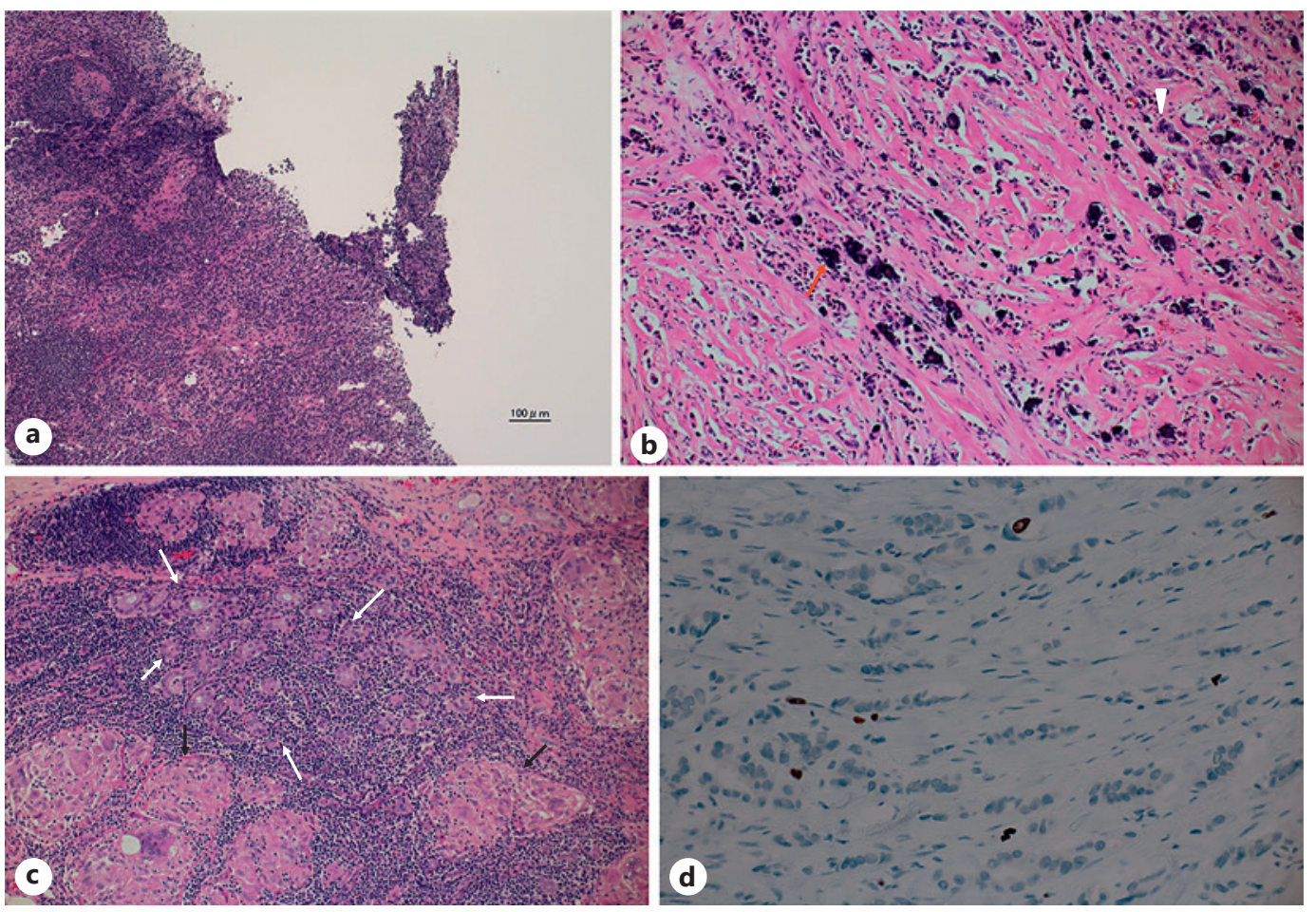

Fig. 3. Pathological findings. a Core needle biopsy of the neck tumor showed massive atypical cells with a large nucleus and scant cytoplasm. b Pathological examination of the neck tumor after the treatment showed massive collagen fibers with microcalcifications (arrow) and scant atypical cancer cells (arrowhead). c Pathological examination of the axillar tumors after the treatment showed massive sarcoid-like reactions (black arrows) and a nominal cancer cell cluster (encompassed by white arrows). d Immunostaining of the resected tumors showed a very low Ki-67 labeling index of 2.5\%.

an immune response site. Unusual anticancer response, that is, long-term minor response followed by very late onset of marked response to the therapy, observed in this neck tumor might also correlate with some kind of immune response.

Compared with MRI, CT, and PET/CT, US is more suitable for frequent evaluation of anticancer effect on the locoregional recurrences but needs careful interpretation. Image diagnosis is overwhelmingly performed by pattern recognition on any diagnostic modality. Due to the discordance between US and PET, we could doubt whether the US evaluation of the anticancer effect of the therapy was correct or not. Breast oncologists, therefore, need to be familiar with the basic principles of ultrasound imaging, especially with the principle of internal echo formation, to avoid both under- and overtreatment.

Surgical intervention in this case does not simply imply the cure of the breast cancer recurrences. No further recurrence, however, has been detected for a year after operation. The neck tumor in this case did not show any characteristics of lymph node structure. We, therefore, could not determine from which mechanisms the neck recurrence developed. Supraclavicular lymph node is now defined as N3c but was once defined as distant metastasis, that is, M1, in the TNM classification. Surgical intervention to this area, therefore, has long been controversial. Oligometastatic breast cancer like this case, however, should be the target of multidisciplinary treatment as curable subjects [5-7].

Similar to cancer cell metastasis and sarcoidosis, sarcoid-like reaction shows fluorodeoxyglucose accumulation on PET/CT $[8,9]$. PET/CT before surgery did not show any avidity in the histologically confirmed massive sarcoid-like reaction. In addition, high Ki-67 labeling 
index on the core needle biopsy specimen decreased to an extremely low level of $2.5 \%$ after a CDK 4/6 inhibitor-containing therapy. These findings suggest that CDK 4/6 inhibitors are possible therapeutic candidates to control sarcoid-like reaction or sarcoidosis. In conclusion, breast oncologists should well understand the basic principles of internal echo formation on US and take the presence of sarcoid-like reaction in the cancer cell clusters into consideration on the therapeutic evaluation of metastatic breast cancer.

\section{Statement of Ethics}

The study is exempt from ethics committee approval due to the nature of reporting based on daily clinical practice. Informed written consent was obtained from the patient for the publication of this case report and any accompanying images.

\section{Conflict of Interest Statement}

The authors have no conflicts of interest to declare.

\section{Funding Sources}

No funding was received for this research.

\section{Author Contributions}

Fujii S. contributed to the design of the report. Oura S. drafted the manuscript. Makimoto S. revised the manuscript. All authors have read and approved the final version of the manuscript.

\section{Data Availability Statement}

All data generated during this study are included in this article. Further enquiries can be directed to the corresponding author.

\section{References}

1 Finn RS, Martin M, Rugo HS, Jones S, Im SA, Gelmon K, et al. Palbociclib and letrozole in advanced breast cancer. N Engl J Med. 2016;375:1925-36.

2 Tomita M, Oura S, Nishiguchi H, Makimoto S. A case of diffuse large B cell lymphoma of the breast with predominantly high-level internal echoes. Case Rep Oncol. 2020 Jan-Apr;13(1):309-13.

3 Kinoshita Y, Ishii H, Eishi Y, Uchida K, Yoshimura M, Iwasaki A, et al. Histological differences between sarcoidosis and lung cancer-related sarcoid reaction. Respir Investig. 2020;58:421-4.

4 Asano S. Granulomatous lymphadenitis. J Clin Exp Hematop. 2012;52:1-16.

5 McDonald ML, Deschamps C, Ilstrup DM, Allen MS, Trastek VF, Pairolero PC. Pulmonary resection for metastatic breast cancer. Ann Thorac Surg. 1994;58:1599-602.

6 Veltri A, Gazzera C, Barrera M, Busso M, Solitro F, Filippini C, et al. Radiofrequency thermal ablation (RFA) of hepatic metastases (METS) from breast cancer (BC): an adjunctive tool in the multimodal treatment of advanced disease. Radiol Med. 2014;119:327-33.

7 Bigorie V, Morice P, Duvillard P, Antoine M, Cortez A, Flejou JF, et al. Ovarian metastases from breast cancer: report of 29 cases. Cancer. 2010;116:799.

\section{Karger's}


8 Chowdhury FU, Sheerin F, Bradley KM, Gleeson FV. Sarcoid-like reaction to malignancy on whole-body integrated (18)F-FDG PET/CT: prevalence and disease pattern. Clin Radiol. 2009;64:675-81.

9 Chida M, Inoue T, Honma K, Murakami K, MD. Sarcoid-like reaction mimics progression of disease after induction chemotherapy for lung cancer. Ann Thorac Surg. 2010;90:2031-3. 